\title{
ÍNDICES PROLIFERATIVOS DO TUMOR VENÉREO CANINO TRANSMISSÍVEL PELAS TÉCNICAS DO CEC E KI-67 NA CITOLOGIA ASPIRATIVA COM AGULHA FINA (Proliferation indexes determination by CEC and Ki-67 in fine needle aspiration cytology of transmissible venereal tumor)
}

\author{
GREATTI, W.F.P. ${ }^{1}$; AMARAL, A.S.'; SILVA, S.B. ${ }^{3}$; GASPAR, L.F.J. \\ BARBISAN, L.F.; ROCHA, N.S. ${ }^{6}$
}

\begin{abstract}
${ }^{1}$ Faculdade de Medicina Veterinária e Zootecnia, UNESP-Botucatu; bolsista de iniciação científica, FADESP; ${ }^{2}$ Curso de Pós-graduação em Medicina Veterinária, doutorado, UNESP-Botucatu. E-mail: anne.am@uol.com.br; ${ }^{3}$ Curso de Pós-graduação em Medicina Veterinária, mestrado, UNESP-Botucatu. E-mail: sabassani@yahoo.com.br;

${ }^{4}$ Curso de Pós-graduação em Medicina Veterinária, doutorado, UNESP-Botucatu. E-mail: ifj.gaspar@uol.com.br;

${ }^{5}$ Departamento de Biologia Celular, Instituto de Biologia, UNESP-Botucatu; ${ }^{6}$ Departamento de Clínica Veterinária, FMVZ-UNESP-Botucatu. E-mail: rochanoeme@fmvz.unesp.br.
\end{abstract}

\begin{abstract}
RESUMO - A citologia aspirativa com agulha fina (CAAF) foi usada como método de colheita de quinze amostras de tumor venéreo transmissível (TVT)em cães, oriundos do atendimento do Hospital Veterinário-FMVZ-UNESP de Botucatu, antes e após o início da quimioterapia. As amostras citológicas foram submetidas ao cálculo do índice mitótico e à avaliação de índices de proliferação por métodos citoquímico (concentração eletrolítica crítica - CEC) e imunocitoquímico (Ki-67, clone MIB-1). Todas as técnicas mostraram-se adequadas para avaliar a taxa proliferativa em amostras citológicas. O CEC permitiu observar que a aplicação do quimioterápico levou a uma redução significativa da proliferação celular. Em vista disso, conclui-se que essa metodologia pode ser aplicada para diagnosticar e monitorar o TVT.
\end{abstract}

Palavras chaves: tumor venéreo transmissível canino, citologia, índices de proliferação, concentração eletrolítica crítica, Ki-67.

\begin{abstract}
Fine needle aspiration biopsy (FNAB) was used to collect fifteen transmissible venereal tumor (TVT) samples from dogs at the Veterinary Hospital - FMVZ - UNESP Botucatu, São Paulo, Brazil, before and after beginning of chemotherapy. The cytological samples were submitted to determination of mitotic and proliferation indexes by means of cytochemical (critical electrolyte concentration - CEC) and immunocytochemical (Ki-67, MIB-1 clone) methods. All the techniques were adequate for the evaluation of proliferation rates displayed by the cytological samples. The CEC allowed the observation that chemotherapy induces to significant reduction in cellular proliferation. Thus, was concluded that this methodology is suitable to perform TVT diagnosis and monitoration.
\end{abstract}

Key words: canine transmissible venereal tumor, cytology, proliferation indexes, critical electrolyte concentration, Ki-67.

\section{Introdução}

O tumor venéreo transmissível (TVT) é uma neoplasia de células redondas transmitida naturalmente aos animais suscetíveis por um mecanismo raro, de transplantação de células tumorais viáveis (CHU et al., 2001). Afeta comumente a genitália externa de cães e é transmitido pelo coito ou hábitos sociais como cheirar e lamber (MACEWEN, 2001). A ocorrência de metástases é mais freqüente em linfonodos, pele e conjuntiva ocular, mas olhos, sistema nervoso central, rins, baço, fígado e vários outros locais também podem ser afetados (DAS e DAS, 2000). 
O TVT pode existir como massa solitária ou lesões múltiplas, em formato de couve-flor, ou como formas pendulares, nodulares, papilares ou multilobulares. O tamanho do tumor pode atingir até 10 a $15 \mathrm{~cm}$. Tem sido reportado que as células de TVT começam a se multiplicar em duas a três semanas após a implantação e alcançar a forma multilobular após um período variável de dois a quatro meses. Apesar de sua potencial natureza maligna, o tumor venéreo responde a diferentes tipos de tratamentos, como radioterapia, crioterapia e quimioterapia, esta última comprovadamente a modalidade terapêutica de maior eficácia, sendo o TVT mencionado como o tumor mais responsivo à quimioterapia em oncologia veterinária (ERÜNAL-MARAL et al., 2000).

Vários estudos têm demonstrado a utilidade da citologia aspirativa com agulha fina (CAAF) no diagnóstico de neoplasias (ROCHA, 1998a,b; ALLEMAN e BAIN, 2000), inclusive o TVT. Nos últimos anos, a freqüência de cães com TVT na rotina do Hospital Veterinário da FMVZ-UNESP de Botucatu tem apresentado crescimento exponencial. Além disso, o comportamento extremamente agressivo desse tumor foge do descrito pela literatura internacional. Estes casos merecem um estudo detalhado (ROCHA, 1998a,b).

A taxa de proliferação de células neoplásicas reflete uma desordem no equilíbrio entre células em divisão, tempo de ciclo celular, diferenciação e senescência, ou seja, é a expressão de mais do que simplesmente replicação celular. A literatura veterinária vem mostrando reiterado interesse na proliferação celular de tumores como uma forma de compreender a biologia tumoral ou de permitir estimativas de prognóstico com ou sem tratamento (MADEWELL, 2001).

Nucléolos sempre foram reconhecidos como uma organela celular que se torna proeminente nas lesões proliferativas, especialmente nas neoplasias malignas. É sabido que células em proliferação ou metabolicamente ativas têm nucléolos mais proeminentes e em maior número (KAMEL et al., 1990). Desta forma, a avaliação do número, forma e tamanho do nucléolo podem ser utilizados como parâmetro de avaliação proliferativa e de progressão neoplásica (BARBISAN et al., 1998). Por meio de técnicas citoquímicas usuais, como a da concentração eletrolítica crítica (CEC), podese obter imagens nucleolares nítidas, possibilitando sua análise morfológica e morfométrica (MELLO et al., 1993).

A capacidade proliferativa aumentada é uma das principais características das células tumorais (PRESTON-MARTIN et al., 1990; CHU et al., 2001). Marcadores de proliferação celular podem ser úteis na avaliação do comportamento biológico da neoplasia, possibilitando inferências com respeito à sensibilidade a regimes de quimioterapia (QUINN e WRIGHT, 1990; GONZALEZ et al., 2000). O total de figuras de mitose (metáfase, anáfase e telófase) é o método tradicionalmente utilizado para estimar a atividade proliferativa das células neoplásicas. Entretanto, como a mitose representa apenas a menor fração do ciclo celular, as células que estiverem nas fases $\mathrm{G}_{1}, \mathrm{~S}$ ou $\mathrm{G}_{2}$ não são contabilizadas, levando a uma sub-estimativa do processo (QUINN e WRIGHT, 1990). Para contornar essa desvantagem, a marcação de antígenos expressos pelas células em proliferação, geralmente por imunoistoquímica, tem sido cada vez mais utilizada em medicina veterinária (MADEWELL, 2001, GUVENC et al., 2002).

O Ki-67 é um anticorpo monoclonal que identifica um antígeno nuclear presente na maioria das células em proliferação. A expressão deste antígeno está intimamente associada com o ciclo celular, podendo ser usado para medir a fração de crescimento das células. Sua expressão ocorre durante a fase $G_{1}$ e progride durante o ciclo celular, alcançando o máximo em $\mathrm{G}_{2}$ e $\mathrm{M}$, diminuindo rapidamente após a mitose, não sendo expresso em $\mathrm{G}_{0}$ (QUINN e WRIGHT, 1990).

Utilizando-se partes recombinantes do antígeno Ki-67 como imunógeno, foram produzidos novos anticorpos monoclonais, designados MIB-1-2-3, dos quais MIB-1 e MIB-3 detectam o mesmo epítopo (ou muito similar) que o anticorpo original Ki-67. Assim, 
Índices proliferativos do tumor venéreo canino transmissível pelas técnicas do CEC e KI-67 na citologia...

o MIB-1 tem-se mostrado um marcador de proliferação celular útil e confiável nos mais diferentes tecidos (IQBAL et al., 2002; HAZAN et al., 2002).

Nossa proposta neste estudo foi verificar a taxa de proliferação celular do tumor venéreo transmissivel em amostras obtidas por citologia antes e durante o tratamento quimioterápico, utilizando métodos de avaliação citológicos, citoquímicos e imunocitoquímicos.

\section{Material e Método}

Foram inclusos no estudo 15 cães, provenientes do atendimento do Hospital Veterinário da FMVZ - UNESP de Botucatu, sem distinção de raça, sexo e idade, com diagnóstico citológico de TVT e sem histórico de quimioterapia prévia.

Foram colhidas duas amostras de cada animal: uma após a confirmação do diagnóstico e outra sete dias após o início do protocolo terapêutico (sulfato de vincristina, na dose de $0,5 \mathrm{mg} / \mathrm{cm}^{2}$ de superfície corporal, via intravenosa obrigatória).

Para a CAAF utilizou-se agulha de $13 \times 4,5 \mathrm{~mm}$ para tumores com até $1 \mathrm{~cm}$ de diâmetro e agulha de $30 \times 7 \mathrm{~mm}$ para tumores maiores. A agulha, acoplada a uma seringa de $10 \mathrm{ml}$, era introduzida na lesão e, enquanto se faziam movimentos em leque, produziase pressão negativa na seringa, amostrando uma ampla área do tumor. Após, a pressão negativa era desfeita, a agulha desconectada da seringa e o seu conteúdo empurrado com ar para lâminas histológicas, onde se confeccionavam os esfregaços. A cada colheita eram obtidos seis esfregaços de cada tumor, dois deles fixados em álcool etílico ainda úmidos e os demais, secos ao ar e fixados em metanol.

Das lâminas fixadas em álcool metílico duas foram coradas pelo Giemsa para avaliação do índice mitótico (IM), obtido pela contagem, em cada lâmina, das células em mitose em um total de 500 células neoplásicas

\footnotetext{
${ }^{1}$ Immunotech, Marseille, France.

2 Dakopatts.

${ }^{3}$ Sigma, St. Louis, EUA.
}

e multiplicado por 100. As outras duas lâminas foram coradas para CEC com uma solução de $0,025 \%$ de azul de toluidina em tampão Mcllvaine pH 4,0 seguida da aplicação de cloreto de magnésio (MELLO et al., 1993). Nestas lâminas fez-se a morfometria nuclear, sob observação em microscópio óptico adaptado ao analisador de imagem KS-300, sob objetiva de $100 x$, consistindo na determinação da área do núcleo e dos nucléolos, em 50 células tumorais escolhidas aleatoriamente, e a razão entre elas (área do núcleo : área do nucléolo). Para análise dos parâmetros de morfometria pela técnica de CEC das amostras de TVT, foi utilizado o teste estatístico de Wilcoxon (ZAR, 1984).

Duas lâminas foram fixadas em álcool etílico $90 \%$, no qual permaneceram até seu processamento pela técnica de imunocitoquímica para a marcação com o anticorpo primário Ki-67, clone MIB-11 diluído a 1:200, seguido de incubação com complexo $A B C^{2}$, por 45 minutos. A revelação foi feita utilizando-se como cromógeno 3,3 diaminobenzidina $^{3}$ (DAB). A contracoloração foi feita com metil green e os esfregaços foram secos em temperatura ambiente e montados com resina sintética. A positividade da reação foi avaliada em microscópio óptico simples, considerando-se positivo a presença de coloração nuclear acastanhada, a despeito da intensidade da coloração. Foram avaliadas 500 células por lâmina, em objetiva de 40x. O índice de proliferação (IP) foi calculado pela divisão do número de células marcadas pelo de células contadas e multiplicadas por 100 .

O índice mitótico e o de proliferação foram estabelecidos somente nas amostras pré-tratamento, já que após a instituição da quimioterapia a população celular obtida na citologia era muito escassa, com células em proliferação em percentual muito baixo.

\section{Resultados e Discussão}

Nossos achados confirmaram o valor da CAAF como método eficiente no 
diagnóstico de TVT (FIGURA 1), pois além de ser pouco invasiva confere de modo preciso e rápido o diagnóstico de neoplasias, conforme já descrito por ROCHA (1998a,b) e ALLEMAN e BAIN (2000). Além disso, a CAAF também permite a confecção de lâminas para estudos complementares. Como desvantagem da técnica, após a instituição da quimioterapia, observou-se que a qualidade do material obtido não era a desejada, já que se obtinha uma pequena quantidade de células íntegras dispersas entre células necróticas e inflamatórias, provavelmente devido à diminuição da população de células viáveis no tumor pela ação do quimioterápico. Isto impediu a estimação dos índices mitótico e de proliferação, uma vez que a quantidade de células viáveis nas amostras foi inferior ao estabelecido para o cálculo destes índices.

FIGURA 1 - CÉLULAS DE TUMOR VENÉREO TRANSMISSÍVEL EM CÃES. OBSERVAR ALTA CELULARIDADE, CITOPLASMA ESCASSO E COM VACÚOLOS, CROMATINA GROSSEIRA E NUCLÉOLOS EVIDENTES E FIGURAS DE MITOSE (SETAS). GIEMSA, 640X.

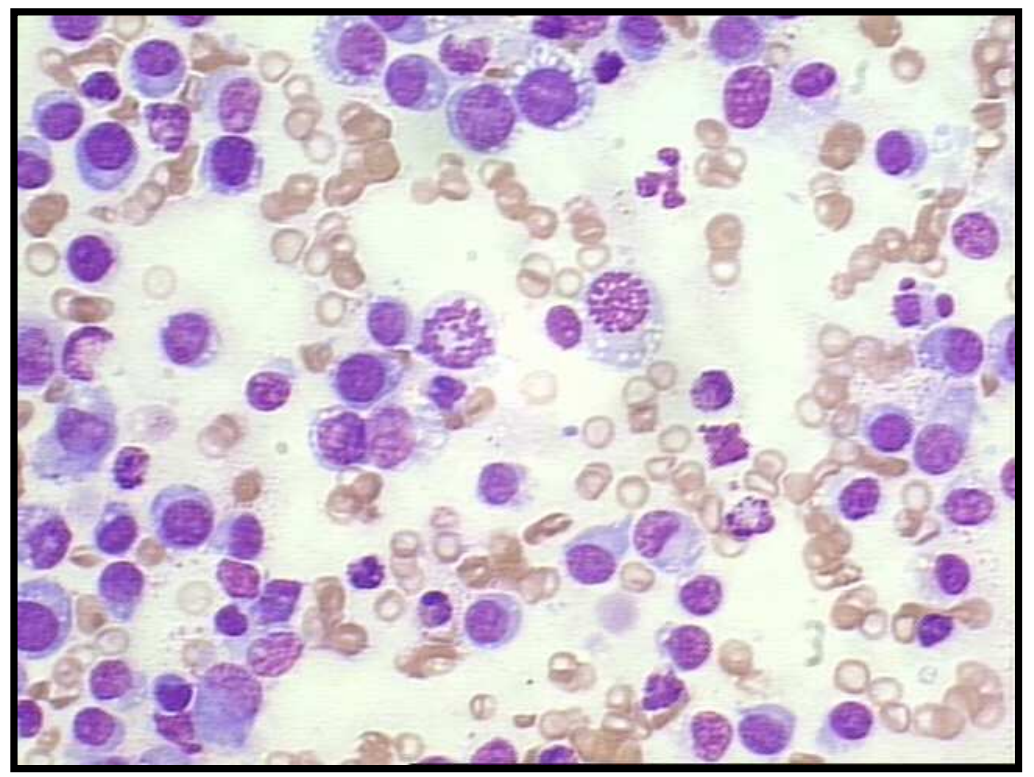

FIGURA 2 - ÁREA NUCLEOLAR E NUCLEAR DE AMOSTRA CITOLÓGICA DE TUMOR VENÉREO TRANSMISSÍVEL EVIDENCIADAS PELO MÉTODO DE CEC EM CÃES (AUMENTO 400X).

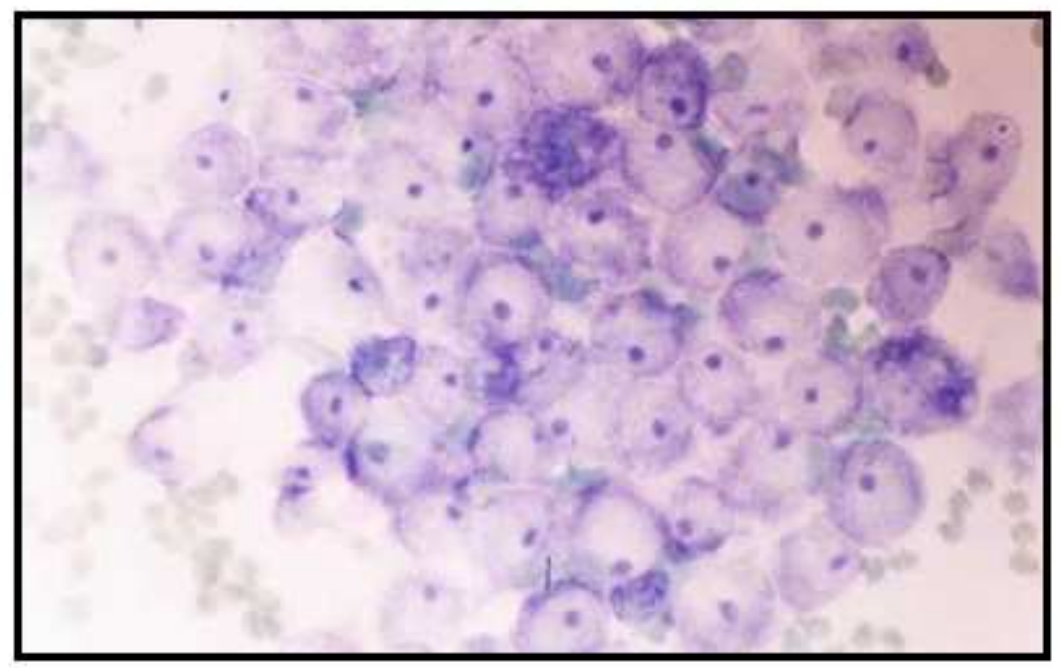


Índices proliferativos do tumor venéreo canino transmissível pelas técnicas do CEC e KI-67 na citologia...

FIGURA 3 - IMUNOMARCAÇÃO PARA KI-67 (CLONE MIB-1) EM CITOLOGIA DE TVT, MOSTRANDO ALTA POSITIVIDADE DAS CÉLULAS NEOPLÁSICAS EM CÃES (640X).

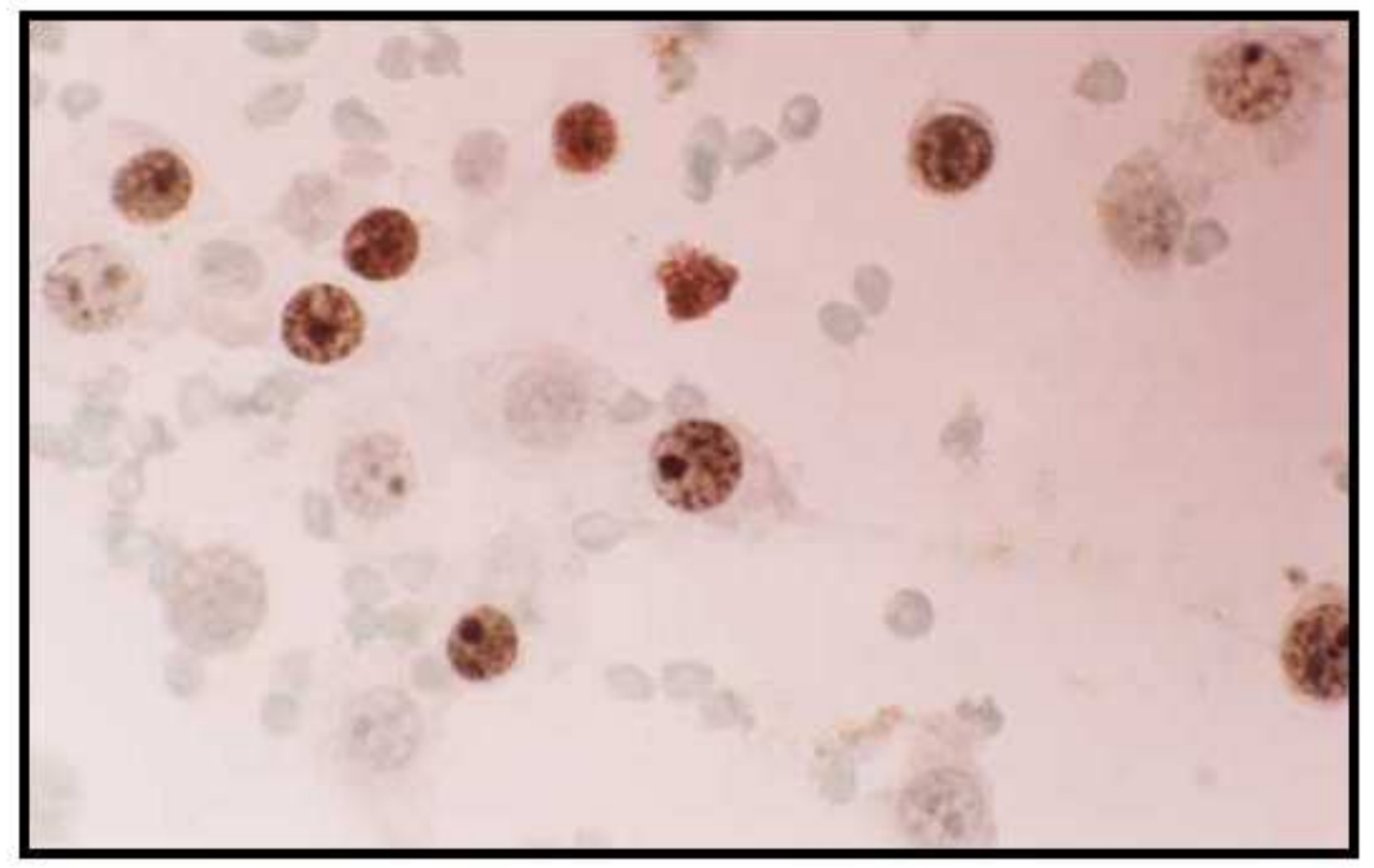

Nas lâminas coradas com o método de CEC (FIGURA 2), obteve-se a morfometria nuclear e nucleolar de cinqüenta células de TVT por amostra, conforme apresentado na TABELA 1.

\section{TABELA 1 - ANÁLISE MORFOMÉTRICA (MÉDIA + DESVIO PADRÃO) DE CÉLULAS DE TUMOR VENÉREO TRANSMISSÍVEL CORADAS PELO MÉTODO DE CONCENTRAÇÃO ELETROLÍTICA CRÍTICA (CEC).}

\begin{tabular}{lcccc}
\hline AMOS TRAS & $\mathrm{n}$ & ÁREA NUCLEOLAR $\left(\mu \mathrm{m}^{2}\right)$ & ÁREA NUCLEAR $\left(\mu \mathrm{m}^{2}\right)$ & Razão \\
\hline Pré-tratamento & 15 & $5,53 \pm 1,87^{\mathrm{a}}$ & $62,31 \pm 29,37^{\mathrm{a}}$ & $0,10 \pm 0,03^{\mathrm{a}}$ \\
Após $1^{\circ}$ tratamento & 09 & $3,16 \pm 1.51^{\mathrm{b}}$ & $49,09 \pm 17,88^{\mathrm{a}}$ & $0,08 \pm 0,03^{\mathrm{a}}$ \\
Nivel de significância & & $\mathrm{p}<0,015$ & $\mathrm{p}<0,295$ & $\mathrm{p}<0,118$ \\
\hline
\end{tabular}

aD Letras diferentes na mesma coluna representam diferença significativa.

Análise estatística realizada somente nos casos que possuíam amostras pareadas.

$\mathrm{Na}$ comparação entre os valores obtidos no pré-tratamento e após a primeira sessão de quimioterapia nos três parâmetros utilizados na análise morfométrica das células de TVT coradas pelo CEC, somente a área nucleolar foi estatisticamente significativa, observando-se uma redução da área dos nucléolos nos animais submetidos ao tratamento. Esse resultado sugere que a quimioterapia induziu à diminuição da atividade nucleolar e, conseqüentemente, da taxa de proliferação celular. Este achado está de acordo com os dados reportados por GONZALEZ et al. (2000), que observaram uma cessação quase total da proliferação celular em casos de TVT com regressão induzida por uma dose de quimioterápico. Já as áreas nucleares das células de TVT coradas pelo CEC foram estatisticamente semelhantes quando se comparou o prétratamento com o pós-tratamento, o que não difere dos resultados encontrados por BARBISAN et al. (1998), que trabalharam com CEC em células epiteliais de mama na mulher.

A utilização do clone MIB-1 para a imunomarcação em amostras obtidas por citologia aspirativa mostrou-se de fácil padronização no TVT, da mesma forma como observado por GUVENC et al. (2002) 
utilizando amostras incluídas em parafina. Foram consideradas positivas para a imunocitoquímica com o Ki-67 aquelas células cujos núcleos apresentavam coloração marrom, independentemente da intensidade do tom, enquanto que as células negativas mostravam núcleo de cor azulada (FIGURA 3). $O$ índice proliferativo (IP) obtido por esta técnica foi de $10,8 \%$ e o índice mitótico (IM) obtido na análise dos esfregaços corados pelo Giemsa foi de $0,73 \%$. A grande diferença entre os valores dos dois parâmetros era esperada, como salientado por QUINN E WRIGHT (1990), visto que a fase de mitose é uma pequena parte do ciclo celular. Apesar da diferença numérica, GONZALEZ et al. (2000) ressaltaram que existe correlação entre a marcação com o Ki-67 e a contagem de mitoses.

No presente trabalho os três métodos de quantificação da proliferação celular mostraram-se eficientes e aplicáveis em amostras citológicas de tumor venéreo transmissível, cada qual com suas vantagens e desvantagens. A CEC é um método de baixo custo e de fácil execução, porém necessita da leitura em um analisador de imagens, o que reduz sua acessibilidade. A imunocitoquímica é uma técnica mais elaborada e o custo dos anticorpos é elevado, comparado a CEC, mas pode ser avaliada em microscopia ótica. Já o índice mitótico é barato e de fácil execução, porém leva a subestimativa da taxa proliferativa, - que limita significativamente sua interpretação. A escolha do método de avaliação de proliferação celular, desta forma, está condicionada à disponibilidade técnica de cada laboratório.

\section{Referências}

ALLEMAN, A.R.; BAIN, P.J. Diagnosing neoplasia: the cytologic criteria for malignancy. Veterinary Medicine, Kansas City, v.95, p.204-248, 2000.

BARBISAN, L.F.; RUSSO, J.; MELLO, M.L.S. Nuclear and nucleolar image analysis of human breast epithelial cells transfored with the C-Ha-ras oncogene. Analytical Cellular Pathology, Amsterdan, v.16, p.193199, 1998.
CHU, R.M.; LIN, C.C.; YANG, S.Y.; HSIAO, Y.W.; HUNG, S.W.; PAO, H.N.; LIAO, K.W. Proliferation characteristics of canine transmissible venereal tumor. Anticancer Research, Atenas, v.21, n.6A, p.4017-4024, 2001.

DAS, U.; DAS, A.K. Review of canine transmissible venereal sarcoma. Veterinary Research Communications, Netherlands, v.24, n.8, p.545-556, 2000.

ERÜNAL-MARAL, N.; FINDIK, M.; ASLAN, S. Use of exfoliative cytology for diagnosis of transmissible venereal tumour and controlling the recovery period in the bitch. Deutsche Tierärztliche Wochenschrift, Hannover, v.107, n.5, p.175-180, 2000.

GONZALEZ, C.M.; GRIFFEY, S.M.; NAYDAN, D.K.; FLORES, E.; CEPEDA, R.; CATTANEO, G.; MADEWELL, B.R. Canine transmissive tumour: a morphological and immunohistochemical study of 11 tumours in growth phase and during regression after chemotherapy. Journal of Comparative Pathology, Edinburg, v.122, n.4, p.241-248, 2000.

GUVENC, T.; HALIGUR, M.; ORMAN, M.N.; HAZIROGLU, R. Mitosis and apoptosis in canine cutaneous histiocytoma and transmissible venereal tumour. Acta Veterinaria Hungarica, Budapeste, v.50, n.3, p.315-321, 2002.

HAZAN, C.; MELZER, K.; PANAGEAS, K.S. Evaluation of the proliferation marker MIB-1 in the prognosis of cutaneous malignants melanoma. Cancer, Hagerstown, v.95, n.3, p.634-640, 2002.

IQBAL, S.; ANDERSON, T.J.; MARSON, L.P. MIB-1 assessments in breast cancers. Breast, Edinburg, v.11, n.3, p.252-256, 2002.

KAMEL, H.M.H.; KIRK, J.; TONER, P.G. Ultrastructural pathology of the nucleus. In: UNDERWOOD,J.C.E. (Ed.) Current opinion pathology: pathology of the nucleus. Berlin: Springer-Verlag, 1990. Chap. 32, p. 1665.

MADEWELL, B.R. Cellular proliferation in tumors: a review of methods, interpretation, and clinical applications. Journal of Veterinary Internal Medicine, Lakewood, v.15, p.334-340, 2001.

MACEWEN, E.G.C. Transmissible venereal tumor. In: WITHROW, S.J.; MACEWEN,E.G.(Eds.) Small animal clinical oncology. 3.ed. Philadelphia: Saunders, 2001. Chap. 29, p.651-656. 
Índices proliferativos do tumor venéreo canino transmissível pelas técnicas do CEC e KI-67 na citologia...

MELLO, M.L.S.; VIDAL, B.C.; DANTAS, M.M.; MONTEIRO, A.L.P. Discrimination of the nucleolus by a critical electrolyte concentration method. Acta Histochemica et Cytochemica, Jena, v.26, p.1-3, 1993.

PRESTON-MARTIN, S.; PIKE, M.C.; ROSS, R.K.; JONES, P.A.; HERDERSON, B.E. Increased cell division as a cause of human cancer. Cancer Research, Baltimore, v.50, p.7415-7421, 1990.

QUINN, C.M.; WRIGHT, N.A. The clinical assessment of proliferation and growth in human

Recebido para publicação: $\quad$ 12/12/2003 Aprovado: tumours: evaluation of methods and application as prognostic variables. Journal of Pathology, Chinchester, v.160, p.93-102, 1990.

ROCHA, N.S. Citologia aspirativa por agulha fina em medicina veterinária (I). Cães e Gatos, São Paulo, n.75, p.15-16, 1998a.

ROCHA, N.S. Citologia aspirativa por agulha fina em medicina veterinária (II). Cães e Gatos, São Paulo, n.79, p.14-16, 1998b.

ZAR, J.H. Bioestatistical analysis. New Jersey: Prentice Hall, 1984. 718p. 